July 2006

\title{
Movement patterns used by the elderly when getting out of bed
}

\author{
Julie Mount \\ Thomas Jefferson University \\ Leslie Kresge \\ Thomas Jefferson University \\ Gabrielle Klaus \\ Thomas Jefferson University \\ Lynn Mann \\ Thomas Jefferson University \\ Caitlin Palomba \\ Thomas Jefferson University \\ Follow this and additional works at: https://jdc.jefferson.edu/ptfp \\ Part of the Physical Therapy Commons \\ Let us know how access to this document benefits you
}

\section{Recommended Citation}

Mount, Julie; Kresge, Leslie; Klaus, Gabrielle; Mann, Lynn; and Palomba, Caitlin, "Movement patterns used by the elderly when getting out of bed" (2006). Department of Physical Therapy Faculty Papers. Paper 2.

https://jdc.jefferson.edu/ptfp/2

This Article is brought to you for free and open access by the Jefferson Digital Commons. The Jefferson Digital Commons is a service of Thomas Jefferson University's Center for Teaching and Learning (CTL). The Commons is a showcase for Jefferson books and journals, peer-reviewed scholarly publications, unique historical collections from the University archives, and teaching tools. The Jefferson Digital Commons allows researchers and interested readers anywhere in the world to learn about and keep up to date with Jefferson scholarship. This article has been accepted for inclusion in Department of Physical Therapy Faculty Papers by an authorized administrator of the Jefferson Digital Commons. For more information, please contact: JeffersonDigitalCommons@jefferson.edu. 


\section{Movement Patterns Used by the Elderly when Getting Out of Bed}

Background and Purpose. Getting out of bed is difficult for $27.6 \%$ of people over 65. The purpose of this study is to describe movement patterns used by older adults for rising from bed. Subjects. 42 healthy subjects between the ages of 65 and 90. Methods. Subjects were videotaped performing 5 trials of getting out of bed. Movement patterns were categorized for each body part. Results. A number of movement patterns occurred that were not reported in younger people. The most frequent movement patterns used were: "roll off" (38.3\%) for the trunk, "double push" (34.7\%) for the far arm, "multi-push" (36.0\%) for the near arm, and "synchronous" (31.2\%) for the legs. Discussion and Conclusion. A variety of movement patterns are used by older people for rising from bed. Movement patterns described in this study can be used as a guide for physical therapists and occupational therapists in teaching ageappropriate strategies for getting out of bed.

(Key words: movement patterns, movement strategies, elderly, bed mobility, getting out of bed, rising from bed)

Julie Mount, PhD, PT: associate Professor of Physical Therapy at Thomas Jefferson University, Philadelphia. Ph: (215) 503-6017, Fax (215) 503-3499, email: julie.mount@ jefferson.edu Leslie Kresge, SPT: Thomas Jefferson University, Philadelphia Gabrielle Klaus, SPT: Thomas Jefferson University, Philadelphia Lynn Mann, SPT: Thomas Jefferson University, Philadelphia Caitlin Palomba, SPT: Thomas Jefferson University, Philadelphia 
On a national survey of people over 65 in the United States, $27.6 \%$ reported having difficulty getting out of bed (Manton, 1998). Many will seek help from physical therapists or occupational therapists in finding a strategy that enables them to get out of bed more easily. However, limited information is available on the movement strategies that older people can use to get out of bed independently.

The range of movement patterns used by adolescents and adults through age 59 for getting out of bed has been well described (Sarnacki, 1985; Ford-Smith \& VanSant, 1993; O'Neil \& VanSant, 1993). Ford-Smith \& VanSant (1993) found that the most commonly used patterns were different among people in their 50's compared to people in their 30's. Alexander and colleagues (1995 \& 1992) conducted studies comparing young and elderly women rising from supine to sitting on the edge of the bed. For this portion of getting out of bed, community dwelling older women were found to push more with their arms compared to healthy young women (Alexander et al, 1992). Healthy older women were also more likely to use a strategy of lateral trunk flexion and rotation with weight bearing through the elbow to increase their base of support (Alexander et al, 1995). These studies by Alexander and colleagues (1995 \& 1992) support our contention that older individuals use different movement patterns for rising from a bed than younger individuals, but these studies did not include men, and they described only a few components of the overall movement strategies.

No research was found that systematically described common strategies used by individuals over the age of 60 for getting out of bed. Collecting descriptive information about how older people get out of bed is the first step towards analyzing relationships among impairments, strategies and functional independence in this activity of daily living, and ultimately identifying more effective interventions for older people who have difficulty getting 
out of bed. Therefore, the purpose of this study is to describe movement patterns used by older people when getting out of bed.

\section{$\underline{\text { Methods }}$}

Subjects

Forty-two healthy elderly individuals, 12 men and 30 women, participated in this study. The mean age was $77.5 \pm 12.5$ and ages ranged from 65 to 90 . Subjects were recruited from an active retirement community in Pennsylvania. Participants were excluded from the study if they had any medical condition that affected the way they got out of bed. These medical conditions included, but were not limited to: cerebral vascular accident, multiple sclerosis, Parkinson's disease, hip or knee replacement in the last 6 months, severe arthritis, and current back pain. Each subject signed an informed consent form before participating in the study. The Institutional Review Board approved the protocol for this study.

\section{Equipment}

Two video cameras mounted on tripods were used to record each subject as the person got out of a standard twin size bed. Wide-angle lenses were used on both cameras so that the full body could be viewed with the cameras fairly close to the bed. The bed was $1.87 \mathrm{~m}$ in length and $0.56 \mathrm{~m}$ in height. A bedside table was available to place near the bed if a subject reported using a table to assist in getting out of bed. A cotton sheet was used to cover the mattress and a pillow was provided at the head of the bed. One video camera faced the side of the bed and the other camera faced the foot of the bed. The side-view camera was placed facing the long axis of the bed, $2.4 \mathrm{~m}$ from the midpoint of the side of the bed. The camera at the foot of the bed was 
positioned facing the short axis of the bed, $1.2 \mathrm{~m}$ from the mid point of the foot of the bed. The center of the camera lenses were $1 \mathrm{~m}$ above the floor. Two erasable boards were placed in front of each camera before each trial began, identifying the subject number and trial number.

\section{Procedure}

Prior to videotaping, a questionnaire was administered about what side of the bed the patient got out of at home and whether any bedside furniture was used. The room was then arranged so that the subject could get out of bed the way the person usually did at home. Subjects were asked to remove their shoes and were taped for five trials of getting in and out of bed. Rest breaks were offered between each trial. The following instructions were given for each trial: "When the camera person says 'Go,' I want you to walk to the bed and get into the bed as you would do at home." A subject who did not spontaneously lie supine was asked if the position in which the person was lying was the position used at home. Subjects who reported that they never lay on their backs at home were allowed to lie in their usual positions. Once the subject was in bed, the following instructions were given: "When the camera person says 'Go' I want you to get out of the bed as you would at home and walk back to the chair and have a seat." One researcher guarded the subjects as necessary to reduce the risk of falling. All subjects were given a rest interval between trials. During the rest break, the researchers changed trial number indicators.

After completing the five trials, subjects were asked if they had ever received any information about ways to get in or out of bed. If they answered yes, they were asked about the source of the information and what they had learned about how to get in or out of bed. 


\section{Data Reduction}

Getting out of bed was analyzed using the categories described by Ford-Smith and VanSant (1993), which divided body actions into four components: 1 . the left (far) upper limb 2. the right (near) upper limb 3. head and trunk 4. the lower limbs (legs). In our study, subjects were allowed to approach the bed from the left or right side depending on their home set-up, making it necessary use to the terms "near" and "far" to describe the arm patterns, as used in the study by Sarnacki (1985) in which these movement categories were first developed.

Two researchers viewed the videotapes recorded from the foot of the bed to analyze the legs and far arm, while two other researchers viewed the videotapes recorded from the side of the bed to describe the movements of the head and trunk and the near arm. The researchers viewed the trials independently to decrease chances of rater bias. The first trial of each subject was viewed and the movements were classified. The second trials were then viewed, and so on until all five trials had been classified. If, during the analysis, the researchers noticed the use of a strategy not included in Ford-Smith's (1993) taxonomy, a description of the strategy was recorded.

After all tapes were reviewed, new categories were developed to reflect the new body movements displayed by the subjects. A new category was developed only if a movement pattern was performed by at least two subjects and for at least three trials. The complete list of categories for the given body part, including any new categories, was taught to two members of the research team who had not previously analyzed that body part. These researchers then viewed the videotapes and categorized each trial using the complete list of categories, including the newly developed categories. 


\section{Data Analysis}

Inter-rater reliability was determined by comparing the percent of exact agreement between the pairs of raters using the complete list of categories. Kappa was then calculated to correct for the potential effect of chance agreements.

The frequency with which each movement pattern of each body region occurred was determined. Chi square tests were used to determine if the frequency of patterns used for each body part for subjects aged 65-90 differed significantly from the frequency of patterns used by individuals aged 50-59 from the study by Ford-Smith and VanSant (1993). Alpha was set at $\mathrm{p}<.05$.

The amount of time it took each subject to go from supine to standing for each trial was determined by digitizing the video so that it could be advanced frame by frame. The video camera recorded at 30 frames per second so the time could be measured to the nearest $1 / 30^{\text {th }}$ of a second. The start time was the frame at which the first movement occurred to initiate getting out of bed and the end time was the frame at which the subject achieved a full upright position.

\section{$\underline{\text { Results }}$}

Forty-two subjects each performed 5 trials of getting out of bed for a total of 210 trials. Due to camera malfunctions, side views of 35 trials were lost and foot views of 11 trials were lost. Therefore, only 175 trials were analyzed for the head and trunk and near arm and 199 trials for the legs and far arm. 


\section{New Movement Pattern Categories}

Fifteen new categories were created. Eight of these new categories were required because in $29.1 \%$ of trials subjects did not lie on their backs. "Legs-first see-saw," "back facing edge move to sit," and "trunk-first see-saw" were created for the head and trunk. Three additional categories were formed for the far arm, "multi-push," "reach across midline and push," and "push from prone or back facing edge." Five new categories were added for the near arm including "multi-push," "back facing edge push," "grasp and multi-push," "grasp leg and push," and "grasp leg and multi-push." Four categories were added for the legs, which included, "synchronous lift with asynchronous foot placement," "reverse step off," "scoot asynchronous," and "prone scoot." Descriptions of these movement patterns are in the Appendix.

\section{Inter-rater Reliability of Categorization}

The measurements of exact agreement between raters was found to be $93 \%$ for head and trunk, $91 \%$ for far arm, $94 \%$ for near arm, and 94\% for legs. The Kappa value was found to be .85 for the head and trunk, .83 for the far arm, .91 for the near arm, and .89 for the legs. These values indicate good reliability (Landis \& Koch, 1977).

\section{Movement Patterns Used by Older People to Get Out of Bed}

The frequency of movement patterns for each body region is presented in table 1 . The frequencies with which movement patterns occurred among the elderly was significantly different from the frequencies with which movement patterns occurred among individuals aged 50 through 59. P values on the $\chi^{2}$ tests were $<.001$ for each of the 4 body parts. 
For our population, the most common movement pattern for the head and trunk was "rolloff" (38\%), and the most common pattern for the far arm was "double push" (35\%). The most frequent pattern for the near arm was "multi-push" (36\%), and "synchronous" (31\%) was observed most often in the legs. The most frequent pattern for the near arm, the "multi-push" was one of the patterns that was not reported among young and middle-aged adults.

\section{Movement Patterns Not Used by Elderly}

Three movement patterns that occurred among healthy middle-aged individuals were not observed in the elderly population. These included "pelvis leading" and "lateral roll" for head and trunk and "lateral lift and push" for far arm.

\section{Most Common Combination of Movement Patterns}

Seventy-five different combinations of movement patterns of the head and trunk, far arm, near arm, and legs were seen in the 170 usable trials. Thirty-five combination patterns were only seen in one trial, while forty combinations were seen in two or more trials. The most common combination of movement patterns included "legs-first see-saw" for the head and trunk, "reach across midline and push" for far arm, "multi-push" for near arm, and "synchronous" for legs. Combinations of movement patterns that occurred in greater than $2.5 \%$ of the trials are included in Table 2 .

\section{Time Required to Get Out of Bed}

The mean time required to go from horizontal on the bed to a full standing position for this healthy older population was 5.3 seconds \pm 2.9 seconds. The range was 2.3 to 17.3 seconds. 
During the process of going from supine to standing, 16 of the 42 subjects, that is $38 \%$ of subjects, paused briefly in sitting for at least some of the trials. Of the 198 trials for which data was available, 67 trials, or $34 \%$ of trials, included a pause.

\section{Variability Within Subjects}

Only two subjects demonstrated the same movement pattern combination across all five trials with no variability; $95 \%$ of these healthy older subjects displayed some variability in the strategies they use to get out of bed across five consecutive trials. Thirteen subjects (31\%) lacked inter-trial variability in the head and trunk, thirteen $(31 \%)$ in the near arm, eleven $(26 \%)$ in the far arm, and eleven (26\%) in the legs.

\section{Bed Mobility Education}

Of the 42 subjects, only seven reported having received information about how to get out of bed. One said she was a nurse and had taught others how to get in and out of bed. Two others had been taught by a nurse: one of these two did not remember what was taught, the other was taught to use the legs as a weight to help get the trunk up. Another subject learned from something she read that she should log roll and push with her hand. During her videotaped trials, she started from sidelying rather than supine and pushed herself up using a seesaw pattern. The same pattern was used by a subject who was taught by a chiropractor to roll on to his side and push with his arms. One subject was taught by a physician to keep her head in line with her body due to problems with dizziness. Only one subject reported having learned from a physical therapist how to get out of bed. She reported that the therapist told her not to twist or turn while standing up, but to first straighten out and stand up without twisting. 


\section{$\underline{\text { Discussion }}$}

This is the first study that systematically describes the range of movement patterns used by healthy individuals over 65 for getting out of bed. Older people use a variety of strategies for getting out of bed. Our research demonstrated that the frequencies of using various strategies for rising from bed is significantly different for people over 65 than for people in their 50's. For example, the most frequent movement pattern for the head and trunk in our study is "roll-off." In contrast, Ford-Smith (1993) identified "come to sit" as the most frequent pattern for this body region among subjects ages 30 to 59. According to Sarnacki's (1985) proposed developmental sequence of the task of rising from bed, the "roll off" pattern is a less developmentally advanced movement pattern than the "come to sit." If Sarnacki's (1985) developmental sequence is correct, then our finding that "roll-off" is more common than "come to sit" for people ages 60-90 suggests that there is a developmental regression (Figure 1). The concept of developmental regression is that in older adulthood movement patterns begin to mimic those that are seen in early childhood (Payne \& Isaacs, 1987). Assuming Sarnacki’s (1985) sequence is valid, then it can be said that middle-aged individuals use a more advanced movement pattern for the head and trunk as compared to the elderly who have begun to regress to a less advanced movement pattern.

The increase in the frequency of the roll-off pattern for our subjects over 65 compared to younger adults is consistent with Alexander and colleagues' (1995) findings that rather than sitting up with straight trunk flexion in the sagittal plane (come to sit) as younger women did, older women were more likely to laterally flex and rotate the trunk (roll-off), leaning on the elbow to broaden their base of support. They proposed two hypotheses for why the roll-off 
pattern was more frequently used among older adults: 1) older individuals may have decreased abdominal strength for performing the come to sit, and may be able to substitute other muscles when using the roll-off; 2) older individuals may have decreased postural stability or increased fear of falling, and the roll-off strategy allowed them to keep an elbow on the mat and maintain a larger base of support than the come to sit strategy.

While many of the older subjects in our study exhibited movement patterns similar to those seen in the Ford-Smith (1993) study of middle-aged adults, several new movement patterns were observed. Hypotheses to explain the new patterns that emerged, fall into 3 major categories: starting position, decreased force-generating capacity, and perceptual issues.

Subjects in the Ford-Smith (1993) study were instructed to lie supine as the starting position for each trial. In our study we were interested in capturing the movement patterns older people actually used for the daily activity of getting out of bed. Consequently, subjects were instructed to lie in bed as they would at home. In $29.1 \%$ of trials, subjects reported that they typically lay in a sidelying position. Some of these subjects reported that they did not sleep on their backs due to difficulty breathing. While they did not provide enough detail to be certain, they may have suffered from supine-dependent sleep apnea (Cartright, 1984). Eight of the 15 new movement patterns were patterns that occurred when the subject started in a sidelying or prone position.

Some of the new categories created for this population reflect techniques that might compensate for low force-generating ability. The new strategies involving multiple pushes in the near and the far arm may occur because some older people cannot generate enough force with each push to translate their body far enough. Grasping the leg to aid in rising from the bed may have been substituting arm strength and leg momentum for weak abdominal muscles or hip 
flexors. Grasping the leg to decrease the difficulty of getting out of bed was previously seen in individuals living in a retirement community who reported having trouble getting out of bed (Alexander et al, 1992). Legs-first seesaw is another strategy in which the momentum of the legs dropping off the edge of the bed may assist in lifting the trunk to an upright position with less activation of trunk muscles.

The "prone scoot", "scoot asynchronous" and some of the multiple pushes occurred when subjects made multiple positional adjustments to get to the edge of the bed. Some subjects first scooted to position the pelvis back toward the center of the bed. Then, as they came to sit, they used multiple pushes with their arms in order to get closer to the edge of the bed. These adjustments could have been due to impaired perception of how close they were or needed to be to the edge of the bed, or may have been caused by a perception that they were at risk of falling if they were too close to the edge during the supine to sit component of the task.

There were several limitations of this study. Subjects were videotaped, which may have increased the threat to external validity. Subjects may perform differently when they are knowingly being recorded and become conscious of how they look. Some subjects may behave in a more flamboyant or self-conscious way in this environment in which they are being observed as compared to their movements when they are at home (Gross, 1991).

The environment in which data were collected was designed to resemble the subject's own bedroom. Data were collected in a bedroom at the retirement facility in which the subjects lived, and the location of the bed and bedside furniture was adjusted according to each subject's description of the setup of his or her own bedroom. However, in this facility an effort was made for the rooms and style of furniture to vary. Some subjects reported that their own beds were a slightly different height, or the mattress was softer or harder. Also, in order to enable the 
researchers to observe the body movements, there were no covers on the beds, and the lights were on. The absence of covers and differences in lighting may have caused subjects to get out of bed differently for this study than they did during the daily task of getting out of bed.

Finally, only 42 subjects participated and all of the subjects were recruited from the same residential retirement facility. Sampling from different environments would allow us to obtain greater external validity, which would permit generalization of our finding to the elderly population as a whole. It is recommended that in the future a larger number of subjects should be recruited within each decade of life between 60 and 90 to reveal the age-related trends across these 3 decades. While the effect sizes of distribution of movement patterns between middle aged individuals and individuals over 60 ranged from medium to large $(0.34-0.67)$, we would expect the sizes of the differences between 2 consecutive decades of life to be smaller. To find a moderate difference in distribution of movement patterns between two decades would require 87 trials (18 subjects) for a power of 0.80 (Portney \& Watkins, 2000). In our current sample of 42 subjects, the only decade for which we had 18 or more subjects was the 70's.

The movement patterns found in this study can be used as a menu for physical therapists and occupational therapists for selecting age-appropriate movement patterns for getting out of bed. Biomechanical analysis of the different strategies is needed to identify the different strength, flexibility, and postural control demands placed on the performers by each strategy. Future research should investigate correlations between impairments and successful use of particular strategies. 


\section{$\underline{\text { Conclusion }}$}

A variety of movement patterns are used by older people for getting out of bed. The most common movement strategies are "roll-off" for the head and trunk, "double push" for the far arm, "multi-push" for the near arm, and "synchronous" for the legs. The average movement time for getting out of bed is 5.3 seconds for healthy individuals over 65. Further research is needed to identify relationships between specific neuromusculoskeletal and cardiopulmonary impairments and successful use of particular movement strategies for getting out of bed. 


\section{References}

Alexander NB, Fry-Welch DK, Marshall, LM et al. (1995). Healthy Young and Old Women Differ in Their Trunk Elevation and Hip Pivot Motions When Rising From Supine to Sitting. Journal of American Geriatric Society, 43(4), 338-343.

Alexander NB, Fry-Welch DK, Ward ME, Folkmier LC (1992). Quantitative Assessment of Bed Rise Difficulty in Young and Elderly Women. Journal of American Geriatric Society, 40(7), 685-691.

Alexander NB, Galecki AT, Nyquist LV et al. (2000). Chair and Bed Rise Performance in ADL-Impaired Congregate Housing Residents. Journal of American Geriatric Society, 48(5), 526-533.

Cartwright RD (1984). Effect of sleep position on sleep apnea severity. Sleep; 7:110-114

Ford-Smith CD, VanSant, AF (1993). Age Differences in Movement Patterns Used to Rise from a Bed in Subjects in the Third through Fifth Decades of Age. Physical Therapy, 73(5), 24-33.

Gross D (1991). Issues Related to Validity of Videotaped Observational Data. Western Journal of Nursing Research, 13(5), 658-663.

Landis JR, Koch GG (1977). The Measurement of Observer Agreement for Categorical Data. Biometrics, 33, 159-174.

Manton KG, Stallard E, Corder LS (1998). The Dynamics of Dimensions of Age-Related Disability 1982 to 1994 in the U.S. Elderly Population. Journal of Gerontology: Biological Sciences, 53A(1), B59-B70.

O’Neil McCoy J, VanSant AF (1993). Movement Patterns of Adolescents Rising from a Bed. Physical Therapy, 73(3), 52-63.

Payne VG, Isaacs LE (1987). Human Motor Development: A Lifespan Approach. Mountain View, California: Mayfield Publishing Co. As cited in Ford-Smith (1993).

Portney LG, Watkins MP (2000). Foundations of Clinical Research: Applications to Practice. $2^{\text {nd }}$ ed. Upper Saddle River, NJ: Prentice-Hall Inc.

Sarnacki S (1985). Rising from Supine on a Bed: A Description of Adult Movement and Hypothesis of Developmental Sequences. Richmond, VA: Medical College of Virginia, Virginia Commonwealth University. Masters of Science Thesis. 
Table 1: Frequency of Movement Patterns

\begin{tabular}{|c|c|c|}
\hline Movement Patterns & $\begin{array}{c}\text { Total } \\
\text { Trials }\end{array}$ & $\begin{array}{c}\text { Percentage } \\
(\%)\end{array}$ \\
\hline \multicolumn{3}{|l|}{ Head and Trunk } \\
\hline Roll Off & 67 & 38.3 \\
\hline Come to Sit & 57 & 32.6 \\
\hline Legs First See-Saw* & 27 & 15.4 \\
\hline Trunk First See-Saw* & 14 & 8.0 \\
\hline Back Facing Edge Move to Sit* & 10 & 5.7 \\
\hline Pelvis Leading & 0 & 0.0 \\
\hline Lateral Roll & 0 & 0.0 \\
\hline \multicolumn{3}{|l|}{ Far Arm } \\
\hline Double Push & 69 & 34.7 \\
\hline Lift and Push & 47 & 23.6 \\
\hline Reach Across Midline and Push* & 47 & 23.6 \\
\hline Multi-push* & 14 & 7.0 \\
\hline Push from Prone or Back Facing Edge* & 11 & 5.5 \\
\hline Lift or Lift and Reach & 7 & 3.5 \\
\hline Push & 4 & 2.0 \\
\hline Lateral Lift and Push & 0 & 0.0 \\
\hline \multicolumn{3}{|l|}{ Near Arm } \\
\hline Multi-Push* & 63 & 36.0 \\
\hline Push & 45 & 25.7 \\
\hline Grasp and Push & 24 & 13.7 \\
\hline Grasp Leg and Push* & 14 & 8.0 \\
\hline Lateral Lift and Push & 11 & 6.3 \\
\hline Back Facing Edge Push* & 9 & 5.1 \\
\hline Grasp Leg and Multi-push* & 6 & 3.4 \\
\hline Grasp Edge of Bed and Multi-push* & 3 & 1.7 \\
\hline \multicolumn{3}{|l|}{ 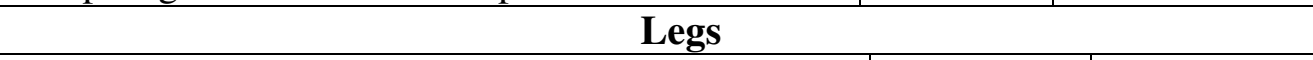 } \\
\hline Synchronous & 62 & 31.2 \\
\hline Asynchronous & 56 & 28.1 \\
\hline Asynchronous with Leg Extension & 42 & 21.1 \\
\hline Step-Off & 11 & 5.5 \\
\hline Scoot Asynchronous* & 9 & 4.5 \\
\hline Synchronous with Asynchronous Foot Placement* & 8 & 4.0 \\
\hline Reverse Step Off* & 6 & 3.0 \\
\hline Prone Scoot* & 5 & 2.5 \\
\hline
\end{tabular}

*indicates new category 
Table 2: Most Common Movement Pattern Combinations

\begin{tabular}{|c|c|c|c|c|c|}
\hline Head \& Trunk & Far Arm & $\begin{array}{c}\text { Near } \\
\text { Arm }\end{array}$ & Legs & Occurrences & Percentage \\
\hline $\begin{array}{c}\text { Legs first see- } \\
\text { saw }\end{array}$ & $\begin{array}{c}\text { Reach across } \\
\text { midline and } \\
\text { push }\end{array}$ & $\begin{array}{c}\text { Multi- } \\
\text { push }\end{array}$ & Synchronous & 12 & $7 \%$ \\
\hline Roll off & Lift and push & Push & Asynchronous & 9 & $5.3 \%$ \\
\hline $\begin{array}{c}\text { Legs First see- } \\
\text { saw }\end{array}$ & $\begin{array}{c}\text { Reach across } \\
\text { midline and } \\
\text { push }\end{array}$ & $\begin{array}{c}\text { Multi- } \\
\text { push }\end{array}$ & Asynchronous & 6 & $3.5 \%$ \\
\hline $\begin{array}{c}\text { Trunk first see- } \\
\text { saw }\end{array}$ & $\begin{array}{c}\text { Reach across } \\
\text { midline and } \\
\text { push }\end{array}$ & $\begin{array}{c}\text { Multi- } \\
\text { push }\end{array}$ & $\begin{array}{c}\text { Scoot } \\
\text { Asynchronous }\end{array}$ & 5 & $2.9 \%$ \\
\hline $\begin{array}{c}\text { Trunk first see- } \\
\text { saw }\end{array}$ & $\begin{array}{c}\text { Reach across } \\
\text { midline and } \\
\text { push }\end{array}$ & $\begin{array}{c}\text { Multi- } \\
\text { push }\end{array}$ & Synchronous & 5 & $2.9 \%$ \\
\hline
\end{tabular}


Figure 1: Age-Related Trend Seen in the Head and Trunk Movement Patterns

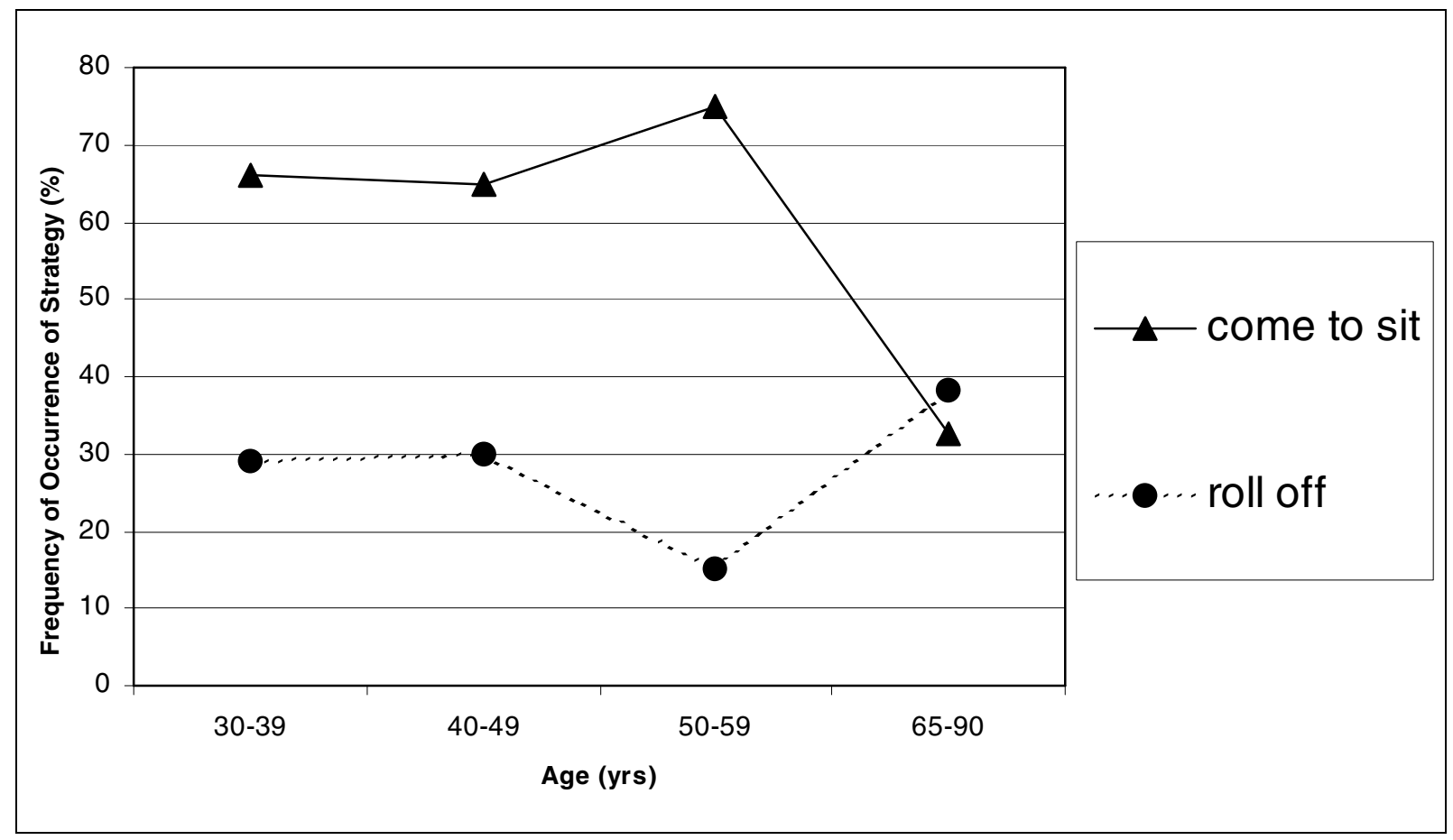




\section{Appendix: Movement Pattern Categories for the Task of Rising from Bed}

\section{Head and Trunk Movement Patterns}

1. Roll Off

During the initial stage of movement (initial $20^{\circ}$ of movement), the head and trunk flex and rotate toward the side-facing position with the weight shifted to one buttock. After the initial stage of movement, the pelvis may drop to a level position while the trunk flexes and comes to the sitting position.

2. Come to sit

During the initial stage of movement (initial $20^{\circ}$ of movement), the head and trunk flex symmetrically by pivoting on both buttocks. After the initial stage of movement, the head and trunk may or may not rotate toward the side-facing position, while the trunk flexes and comes to the sitting position.

3. Trunk first see-saw

Position in sidelying facing the edge of the bed, the trunk laterally flexes and comes to sit then the pelvis drops over the edge of the bed.

4. Legs first see-saw

Position in sidelying facing the edge of the bed, the upper pelvis drops toward the foot of the bed as the trunk laterally flexes and comes to the sitting position.

5. Back facing edge move to sit

Positioned in prone or sidelying with the back facing the edge of the bed, the trunk laterally flexes and rotates pivoting on one or both buttocks as legs drop off the bed.

6. Pelvis Leading

The pelvis rotates to sidelying first. The upper side of the pelvis drops to the bed and the trunk lifts and turns toward the side-facing position.

7. Lateral Roll

The head and trunk turn keeping the shoulders in line with the hips into the sidelying position. The legs drop off the bed as the trunk laterally flexes or rotates to come to sitting position. 


\section{Far Arm Movement Patterns}

1. Push

The arm pushes into the bed and extends until the hand or elbow is the only part of the arm remaining on the bed.

2. Double Push

The arm either lifts toward the head of the bed and pushes or pushes into the bed without lifting. The arm extends until the hand or elbow is the only part of the limb remaining on the bed. The hand or elbow lifts. The hand is placed on the bed, usually near the edge, and pushes to stand.

3. Lift and Push

The arm lifts off the bed and may cross body. The hand is placed on the bed on the far side of the body at some point between the starting position and the edge of the bed and pushes.

4. Lift or Lift and Reach

The arm lifts off the bed and may reach across body but does not push.

5. Multi-push

The arm pushes into the bed and extends until the hand or elbow is the only part of the arm remaining on the bed. The hand then pushes repeatedly at various points on the far side of the body between the starting position and the edge of the bed. The hand may or may not push to stand.

6. Reach Across Midline and Push

The arm may or may not push from the supine position. In the sidelying position facing the edge of the bed, the arm is placed anterior to the trunk on the bed. The hand may or may not grasp the edge of the bed. The arm pushes into the bed and extends the elbow. The arm then lifts, moves across the body, and is placed on the bed next to the far side of the trunk. The hand then pushes to stand.

7. Push from Prone or Back-Facing-Edge

Positioned in prone or sidelying with the back facing the edge of the bed, the arm remains anterior to the trunk and may or may not cross midline. The arm pushes into the bed, extending the elbow until only the hand remains on the bed. The hand may push repeatedly until the weight is transferred to the feet. The trunk may rotate and the hand may push to stand. 
8. Lateral Lift and Push

The upper arm lifts or slides on the supporting surface toward the head of the bed. The arm is placed on the bed and pushes. The arm extends until the hand is the only part of the arm remaining on the bed.

\section{Near Arm Movement Patterns}

1. Lateral lift and Push

The upper arm lifts or slides on the supporting surface toward the head of the bed. The arm is placed on the bed and pushes until the arm is extended or nearly extended and the hand is the only part of the arm remaining on the bed.

2. Grasp and Push

The hand grasps the edge of the bed and the entire arm or some part of it pushes down onto the bed.

3. Push

The arm or some part of it pushes into the bed.

4. Multi-push

The arm pushes from the original position into the bed. The hand then pushes more than one time at various points on the near side of the body. The hand is lifted and placed on the edge of the bed. The hand may or may not push to stand.

5. Back Facing Edge Push

Positioned in prone or sidelying with the back facing the edge of the bed, the arm pushes from the original position into elbow extension and slight shoulder abduction. The hand may or may not push to stand.

6. Grasp and Multi-push

The hand grasps the edge of the bed and the entire arm or some part of it pushes more than one time. The arm may or may not push to stand.

7. Grasp Leg and Push

The hand grasps the leg and the entire arm or some part of it pushes down onto the bed.

8. Grasp Leg and Multi-Push

The hand grasps the leg and the entire arm or some part of it pushes more than one time. The arm may or may not push to stand. 


\section{Leg Movement Patterns}

1. Step Off

The far leg is lifted higher off the bed than the near leg. The near foot comes out of the bed and hits the floor first, followed by the far foot. The near leg may or may not extend before far leg.

2. Asynchronous with Leg Extension

The legs are lifted asynchronously and the far leg may push on the bed before lifting. The thighs remain parallel moving out of bed while the knee of the far leg remains more extended than knee of near leg. The far foot lands on the floor in front of the near foot.

3. Asynchronous

The legs are lifted asynchronously off the bed. The far leg may push on the bed and be medially rotated before lifting. The thighs are parallel as they move out of the bed and the legs are parallel as they descend to the floor with both feet reaching the floor simultaneously.

4. Synchronous

The legs are simultaneously lifted or slid out of the bed. The thighs move parallel out of the bed and both feet reach the floor simultaneously.

5. Synchronous Lift with Asynchronous Foot Placement

The legs are simultaneously lifted or slid out of the bed. The thighs move parallel out of the bed however, the near foot hits the floor first followed by the far foot.

6. Reverse Step Off

Positioned in prone or sidelying with the back facing the edge of the bed, the hips extend asynchronously toward the edge of the bed with one leg stepping off the bed and reaching the floor first. The other foot then reaches the floor and the knees extend.

7. Scoot Asynchronous

The legs push into the bed to move closer to the edge of the bed. The legs move asynchronously off the bed and both feet reach the floor simultaneously.

8. Prone Scoot

Positioned in prone the near leg's knee slides to the side and the pelvis rotates to the sidelying position. The legs may be lifted asynchronously or synchronously and both feet reach the floor simultaneously. 